\title{
Extended First-Order Logic
}

\author{
Chad E. Brown and Gert Smolka \\ Saarland University
}

June 5, 2009

\begin{abstract}
We consider the EFO fragment of simple type theory, which restricts quantification and equality to base types but retains lambda abstractions and higher-order variables. We show that this fragment enjoys the characteristic properties of first-order logic: complete proof systems, compactness, and countable models. We obtain these results with an analytic tableau system and a concomitant model existence lemma. All results are with respect to standard models. The tableau system is well-suited for proof search and yields decision procedures for substantial fragments of EFO.
\end{abstract}

\section{Introduction}

First-order logic can be considered as a natural fragment of Church's type theory [1]. In this paper we exhibit a larger fragment of type theory, called EFO, that still enjoys the characteristic properties of first-order logic: complete proof systems, compactness, and countable models. EFO restricts quantification and equality to base types but retains lambda abstractions and higher-order variables. Like type theory, EFO has a type $o$ of truth values and admits functions that take truth values to individuals. Such functions are not available in firstorder logic. A typical example is a conditional $C: o \iota \iota$ taking a truth value and two individuals as arguments and returning one of the individuals. Here is a valid EFO formula that specifies the conditional and states one of its properties:

$$
(\forall x y . C \perp x y=y \wedge C \top x y=x) \rightarrow C(x=y) x y=y
$$

The starting point for EFO is an analytic tableau system derived from Brown's Henkin-complete cut-free one-sided sequent calculus for extensional type theory [2]. The tableau system is well-suited for proof search and yields decision procedures and the finite model property for three substantial fragments of EFO: lambda-free formulas (e.g., $p a \rightarrow p b \rightarrow p(a \wedge b)$ ), Bernays-Schönfinkel-Ramsey 
formulas [4], and equations between pure lambda terms (terms not involving type $o$ ). The decidability and finite model results are mostly known, but it is remarkable that we obtain them with a single tableau system.

The proofs of the main results follow the usual development of first-order logic [9, 5], which applies the abstract consistency technique to a model existence lemma for the tableau system (Hintikka's Lemma). Due to the presence of higher-order variables and lambda abstractions, the proof of the EFO model existence lemma is much harder than it is for first-order logic. We employ the possible-values technique [8], which has been used in [2] to obtain Henkin models, and in [3] to obtain standard models. We generalize the model existence theorem such that we can obtain countable models using the abstract consistency technique.

In a preceding paper [3], we develop a tableau-based decision procedure for the quantifier- and lambda-free fragment of EFO and introduce the possiblevalues-based construction of standard models. In this paper we extend the model construction to first-order quantification and lambda abstraction. We introduce a novel subterm restriction for the universal quantifier and employ an abstract normalization operator, both essential for proof search and decision procedures.

\section{Basic Definitions}

Types $(\sigma, \tau, \mu)$ are obtained with the grammar $\tau::=o|\iota| \tau \tau$. The elements of $o$ are the two truth values, $\iota$ is interpreted as a nonempty set, and a function type $\sigma \tau$ is interpreted as the set of all total functions from $\sigma$ to $\tau$. For simplicity, we provide only one sort $\iota$. Everything generalizes to countably many sorts.

We distinguish between two kinds of names, called constants and variables. Every name comes with a type. We assume that there are only countably many names, and that for every type there are infinitely many variables of this type. If not said otherwise, the letter $a$ ranges over names, $c$ over constants, and $x$ and $y$ over variables.

Terms $(s, t, u, v)$ are obtained with the grammar $t::=a|t t| \lambda x . t$ where an application st is only admitted if $s: \tau \mu$ and $t: \tau$ for some types $\tau$ and $\mu$. Terms of type $o$ are called formulas. A term is lambda-free if it does not contain a subterm that is a lambda abstraction. We use $\mathcal{N} s$ to denote the set of all names that have a free occurrence in the term $s$.

We assume that $\perp: o, \neg: o o, \wedge: o o o,=_{\sigma}: \sigma \sigma o$, and $\forall_{\sigma}:(\sigma o) o$ are constants for all types $\sigma$. We write $\forall x$.s for $\forall_{\sigma}(\lambda x . s)$. An interpretation is a function $\mathcal{I}$ that is defined on all types and all names and satisfies the following conditions:

- $\mathcal{L} o=\{0,1\}$ 
- $\mathcal{I}(\sigma \tau)$ is the set of all total functions from $\mathcal{I} \sigma$ to $\mathcal{I} \boldsymbol{T}$

- $\mathcal{I} \perp=0$

- $\mathcal{I}(\neg), \mathcal{I}(\wedge), \mathcal{I}\left(=_{\sigma}\right)$, and $\mathcal{I}\left(\forall_{\sigma}\right)$ are the standard interpretations of the respective logical constants.

We write $\hat{\mathcal{I}} s$ for the value the term $s$ evaluates to under the interpretation $\mathcal{I}$. We say that an interpretation $\mathcal{I}$ is countable [finite] if $\mathcal{I} \iota$ is countable [finite]. An interpretation $\mathcal{I}$ is a model of a set $A$ of formulas if $\hat{\mathcal{I}} s=1$ for every formula $s \in A$. A set of formulas is satisfiable if it has a model.

The constants $\perp, \neg, \wedge,=_{\iota}$, and $\forall_{\iota}$ are called EFO constants. An EFO term is a term that contains no other constants but EFO constants. We write $\mathrm{EFO}_{\sigma}$ for the set of all EFO terms of type $\sigma$. For simplicity, we work with a restricted set of EFO constants. Everything generalizes to the remaining propositional constants, the identity $=_{o}$, and the existential quantifier $\exists_{\iota}$.

\section{Normalization}

We assume a normalization operator [] that provides for lambda conversion. The normalization operator [] must be a type preserving total function from terms to terms. We call $[s]$ the normal form of $s$ and say that $s$ is normal if $[s]=s$.

There are several possibilities for the normalization operator []: $\beta$-, long $\beta$-, or $\beta \eta$-normal form, all possibly with standardized bound variables [7]. We will not commit to a particular operator but state explicitly the properties we require for our results. To start, we require the following properties:

$\mathrm{N} 1 \quad[[s]]=[s]$

N2 $[[s] t]=[s t]$

N3 $\left[a s_{1} \ldots s_{n}\right]=a\left[s_{1}\right] \ldots\left[s_{n}\right]$ if the type of $a s_{1} \ldots s_{n}$ is $o$ or $\iota$

N4 $\hat{\mathfrak{T}}[s]=\hat{\mathfrak{T}} s$

Note that $a$ ranges over names and $\mathcal{I}$ ranges over interpretations. N3 also applies for $n=0$.

Proposition 3.1 A term $a s_{1} \ldots s_{n}$ of type $o$ or $\iota$ is normal iff $s_{1}, \ldots, s_{n}$ are normal.

Proof Follows from N3.

We need further properties of the normalization operator that can only be expressed with substitutions. A substitution is a type preserving partial function from variables to terms. If $\theta$ is a substitution, $x$ is a variable, and $s$ is a term that has the same type as $x$, we use $\theta_{S}^{x}$ to denote the substitution that agrees 


$$
\begin{gathered}
\mathcal{T}_{\neg} \frac{s, \neg s}{\perp} \quad \mathcal{T}_{\neg\urcorner} \frac{\neg \neg s}{s} \quad \mathcal{T}_{\wedge} \frac{s \wedge t}{s, t} \quad \mathcal{T}_{\neg \wedge} \frac{\neg(s \wedge t)}{\neg s \mid \neg t} \\
\mathcal{T}_{\forall} \frac{\forall_{\iota} s}{[s t]} t: \iota \quad \mathcal{T}_{\neg \forall} \frac{\neg \forall_{l} s}{\neg[s x]} x: \iota \text { fresh } \\
\mathcal{T}_{\text {MAT }} \frac{x s_{1} \ldots s_{n}, \neg x t_{1} \ldots t_{n}}{s_{1} \neq t_{1}|\cdots| s_{n} \neq t_{n}} \quad \mathcal{T}_{\text {DEC }} \frac{x s_{1} \ldots s_{n} \neq{ }_{\imath} x t_{1} \ldots t_{n}}{s_{1} \neq t_{1}|\cdots| s_{n} \neq t_{n}} \\
\mathcal{T}_{\text {BE }} \frac{s \neq s t}{s, \neg t \mid \neg s, t} \quad \mathcal{T}_{\mathrm{FE}} \frac{s \neq \sigma \tau}{[s x] \neq[t x]} x: \sigma \text { fresh } \\
\mathcal{T}_{\text {CON }} \frac{s \neq t, u \neq, v}{s \neq u, t \neq u \mid s \neq v, t \neq v}
\end{gathered}
$$

Figure 1: Tableau system $\mathcal{T}$

everywhere with $\theta$ but possibly on $x$ where it yields $s$. We assume that every substitution $\theta$ can be extended to a type preserving total function $\hat{\theta}$ from terms to terms such that the following conditions hold:

S1 $\hat{\theta} a=$ if $a \in \operatorname{Dom} \theta$ then $\theta a$ else $a$

S2 $\hat{\theta}(s t)=(\hat{\theta} s)(\hat{\theta} t)$

S3 $[(\hat{\theta}(\lambda x . s)) t]=\left[\widehat{\theta_{t}^{x}} s\right]$

S4 $[\hat{\emptyset} s]=[s]$

S5 $\mathcal{N}[s] \subseteq \mathcal{N} s$ and $\mathcal{N}(\hat{\theta} s) \subseteq \bigcup\{\mathcal{N}(\hat{\theta} a) \mid a \in \mathcal{N} s\}$

Note that $a$ ranges over names and that $\emptyset$ (the empty set) is the substitution that is undefined on every variable.

\section{Tableau System}

The results of this paper originate with the tableau system $\mathcal{T}$ shown in Figure 1. The rules in the first two lines of Figure 1 are the familiar rules from first-order logic. The rules in the third and fourth line deal with embedded formulas. The mating rule $\mathcal{T}_{\text {MAT }}$ decomposes complementary atomic formulas by introducing disequations that confront corresponding subterms. Disequations can be further decomposed with $\mathcal{T}_{\text {DEC }}$. Embedded formulas are eventually raised to the top level by Rule $\mathcal{T}_{\mathrm{BE}}$, which incorporates Boolean extensionality. Rule $\mathcal{T}_{\mathrm{FE}}$ in- 
corporates functional extensionality. It reduces disequations at functional types to disequations at lower types. The confrontation rule $\mathcal{T}_{\text {CoN }}$ deals with positive equations at type $\iota$. A discussion of the confrontation rule can be found in [3]. The tableau rules are such that they add normal formulas if they are applied to normal formulas.

Example 4.1 The following tableau refutes the formula $p f \wedge \neg p(\lambda x . \neg \neg f x)$ where $p:(\iota \circ) o$ and $f: \iota \circ$.

\begin{tabular}{|c|c|}
\hline \multicolumn{2}{|c|}{$\begin{array}{c}p f \wedge \neg p(\lambda x . \neg \neg f x) \\
p f, \neg p(\lambda x . \neg \neg f x) \\
f \neq(\lambda x . \neg \neg f x) \\
f x \neq \neg \neg f x\end{array}$} \\
\hline $\begin{array}{c}f x, \neg \neg \neg f x \\
\neg f x \\
\perp\end{array}$ & $\begin{array}{c}\neg f x, \neg \neg f x \\
\perp\end{array}$ \\
\hline
\end{tabular}

The rules used are $\mathcal{T}_{\wedge}, \mathcal{T}_{\mathrm{MAT}}, \mathcal{T}_{\mathrm{FE}}, \mathcal{T}_{\mathrm{BE}}, \mathcal{T}_{\neg \neg}$, and $\mathcal{T}_{\neg}$.

\section{Evidence}

A quasi-EFO formula is a disequation $s \neq_{\sigma} t$ such that $s$ and $t$ are EFO terms and $\sigma \neq \imath$. Note that the rules $\mathcal{T}_{\mathrm{MAT}}$ and $\mathcal{T}_{\mathrm{DEC}}$ may yield quasi-EFO formulas when they are applied to EFO formulas. A branch is a set of normal formulas $s$ such that $s$ is either EFO or quasi-EFO.

A term $s: \iota$ is discriminating in a branch $A$ if $A$ contains a disequation $s \neq t$ or $t \neq s$ for some term $t$. We use $\mathcal{D} A$ to denote the set of all terms that are discriminating in a branch $A$.

Proposition 5.1 Let $A$ be a branch. Then all terms in $\mathcal{D} A$ are normal.

Proof Follows with N3 since a branch contains only normal formulas.

A branch $E$ is evident if it satisfies the evidence conditions in Figure 2, The evidence conditions correspond to the tableau rules and are designed such that a branch that is closed under the tableau rules and does not contain $\perp$ is evident. Note that the evidence conditions require less than the tableau rules:

1. $\mathcal{E}_{\neg}$ is restricted to variables.

2. $\mathcal{E}_{\forall}$ requires less instances than $\mathcal{T}_{\forall}$ admits.

3. $\mathcal{E}_{\neg \forall}$ admits all EFO terms as witnesses.

4. $\mathcal{E}_{\neq}$is restricted to type $\iota$. 


$$
\begin{aligned}
& \mathcal{E}_{\perp} \perp \text { is not in } E . \\
& \mathcal{E}_{\neg} \quad \text { If } \neg x \text { is in } E \text {, then } x \text { is not in } E \text {. } \\
& \mathcal{E}_{\neg \neg} \quad \text { If } \neg \neg S \text { is in } E \text {, then } s \text { is in } E \text {. } \\
& \mathcal{E}_{\wedge} \quad \text { If } s \wedge t \text { is in } E \text {, then } s \text { and } t \text { are in } E \text {. } \\
& \mathcal{E}_{\neg \wedge} \quad \text { If } \neg(s \wedge t) \text { is in } E \text {, then } \neg s \text { or } \neg t \text { is in } E \text {. } \\
& \mathcal{E}_{\forall} \quad \text { If } \forall_{l} s \text { is in } E \text {, then [st] is in } E \text { for all } t \in \mathcal{D} E \text {, } \\
& \text { and }[s t] \text { is in } E \text { for some } t \in \mathrm{EFO}_{\iota} \text {. } \\
& \mathcal{E}_{\neg \forall} \quad \text { If } \neg \forall_{\iota} s \text { is in } E \text {, then } \neg[s t] \text { is in } E \text { for some } t \in \mathrm{EFO}_{\iota} \text {. } \\
& \mathcal{E}_{\text {MAT }} \quad \text { If } x s_{1} \ldots s_{n} \text { and } \neg x t_{1} \ldots t_{n} \text { are in } E \text { where } n \geq 1 \text {, } \\
& \text { then } s_{i} \neq t_{i} \text { is in } E \text { for some } i \in\{1, \ldots, n\} \text {. } \\
& \mathcal{E}_{\mathrm{DEC}} \quad \text { If } x s_{1} \ldots s_{n} \neq{ }_{\iota} x t_{1} \ldots t_{n} \text { is in } E \text { where } n \geq 1 \text {, } \\
& \text { then } s_{i} \neq t_{i} \text { is in } E \text { for some } i \in\{1, \ldots, n\} \text {. } \\
& \mathcal{E}_{\neq} \quad \text { If } s \neq_{\iota} t \text { is in } E \text {, then } s \text { and } t \text { are different. } \\
& \mathcal{E}_{\mathrm{BE}} \quad \text { If } s \neq_{o} t \text { is in } E \text {, then either } s \text { and } \neg t \text { are in } E \text { or } \neg s \text { and } t \text { are in } E \text {. } \\
& \mathcal{E}_{\mathrm{FE}} \quad \text { If } s \neq_{\sigma \tau} t \text { is in } E \text {, then }[s x] \neq[t x] \text { is in } E \text { for some variable } x \text {. } \\
& \mathcal{E}_{\mathrm{CoN}} \quad \text { If } s={ }_{\iota} t \text { and } u \neq{ }_{\iota} v \text { are in } E \text {, } \\
& \text { then either } s \neq u \text { and } t \neq u \text { are in } E \text { or } s \neq v \text { and } t \neq v \text { are in } E \text {. }
\end{aligned}
$$

Figure 2: Evidence conditions

In $\$ 7$ we will show that every evident branch is satisfiable. In $\$ 9$ we will prove the completeness of a tableau system $\mathcal{R}$ that restricts the rule $\mathcal{T}_{\forall}$ as suggested by the evidence condition $\mathcal{E}_{\forall}$.

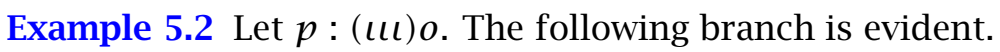

$$
p(\lambda x y \cdot x), \quad \neg p(\lambda x y \cdot y), \quad(\lambda x y \cdot x) \neq(\lambda x y \cdot y), \quad(\lambda y \cdot x) \neq(\lambda y \cdot y), \quad x \neq y
$$

Example 5.3 Let $f: \iota 0$ and $g: o \iota$ be variables. The branch

$$
f\left(g\left(\forall_{l} f\right)\right), \neg f\left(g\left(\forall_{l} f\right)\right), \quad g\left(\forall_{l} f\right) \neq_{l} g\left(\forall_{l} f\right), \quad \forall_{l} f \neq_{o} \forall_{l} f, \quad \forall_{l} f, \neg \forall_{l} f
$$

is unsatisfiable and satisfies all evidence conditions but $\mathcal{E}_{\neq}$. Note that the mating rule does not apply to $\forall_{\iota} f$ and $\neg \forall_{\iota} f$ since $\forall_{\iota}$ is a constant and not a variable. 


\section{Carriers}

A carrier for an evident branch $E$ consists of a set $D$ and a relation $\triangleright_{\iota} \subseteq \mathrm{EFO}_{\iota} \times D$ such that certain conditions are satisfied. We will show that every evident branch has carriers, and that for every carrier $\left(D, \triangleright_{l}\right)$ for an evident branch $E$ we can obtain a model $\mathcal{I}$ of $E$ such that $\mathcal{I} \iota=D$ and $s \triangleright_{\imath} \hat{\mathcal{I}} s$ for all $s \in \mathrm{EFO}_{\iota}$. We call $\triangleright_{\iota}$ a possible-values relation and read $s \triangleright_{1} a$ as $s$ can be $a$. Given $s \triangleright_{1} a$, we say that $a$ is a possible value for $s$.

We assume that some evident branch $E$ is given. We say that a set $T \subseteq \mathrm{EFO}_{\iota}$ is compatible if there are no terms $s, t \in T$ such that $([s] \neq[t]) \in E$. We write $s \sharp t$ if $E$ contains the disequation $s \neq t$ or $t \neq s$.

Let a non-empty set $D$ and a relation $\triangleright_{\iota} \subseteq \mathrm{EFO}_{\iota} \times D$ be given. For $T \subseteq \mathrm{EFO}_{\iota}$ and $a \in D$ we write $T \triangleright_{\iota} a$ if $t \triangleright_{\iota} a$ for every $t \in T$. For all terms $s, t \in \mathrm{EFO}_{\iota}$, all values $a, b \in D$, and every set $T \subseteq \mathrm{EFO}_{\text {}}$ we require the following properties:

B1 $s \triangleright_{\imath} a$ iff $[s] \triangleright_{\imath} a$.

B2 $T$ compatible iff $T \triangleright_{\iota} a$ for some $a \in D$.

B3 If $\left(s={ }_{l} t\right) \in E$ and $s \triangleright_{\iota} a$ and $t D_{\iota} b$, then $a=b$.

B4 For every $a \in D$ either $t \triangleright_{\text {। }} a$ for some $t \in \mathcal{D} E$ or $t \triangleright_{\text {। }} a$ for every $t \in \mathrm{EFO}_{\text {เ }}$.

Given an evident branch $E$, a carrier for $E$ is a pair $\left(D, \triangleright_{\iota}\right)$ as specified above.

\subsection{Quotient-Based Carriers}

A branch $A$ is complete if for all $s, t \in \mathrm{EFO}_{\iota}$ either $[s=t]$ is in $A$ or $[s \neq t]$ is in $A$. We will show that complete evident branches have countable carriers that can be obtained as quotients of $\mathrm{EFO}_{\iota}$ with respect to the equations contained in the branch.

Let $E$ be a complete evident branch in the following. We write $s \sim t$ if $s$ and $t$ are EFO terms of type $\iota$ and $\left[s={ }_{\iota} t\right] \in E$. We define $\tilde{s}:=\{t \mid t \sim s\}$ for $s \in \mathrm{EFO}_{\iota}$.

Proposition 6.1 For all $s, t \in \mathrm{EFO}_{\iota}: s \nsim t$ iff $[s \neq t] \in E$.

Proof One direction is obvious, the other follows with N3.

Proposition $6.2 \sim$ is an equivalence relation on $\mathrm{EFO}_{\iota}$.

Proof We show symmetry by contradiction. Let $s \sim t$ and assume $t \times s$. Then $[s=t]$ and $[t \neq s]$ are in $E$. By N3 we know that $[s]=[t]$ and $[t] \neq[s]$ are in $E$. By $\mathcal{E}_{\text {CoN }}$ either $[t] \neq[t]$ or $[s] \neq[s]$ is in $E$. Contradiction by $\mathcal{E}_{\neq}$. Reflexivity and transitivity follow with similar arguments.

Proposition 6.3 Let $T \subseteq \mathrm{EFO}_{\iota}$. Then $T$ is compatible iff $s \sim t$ for all $s, t \in T$. 
Proof By definition and N3, $T$ is compatible if $[s \neq t] \notin E$ for all $s, t \in T$. The claim follows with Proposition 6.1.

Lemma 6.4 Every complete evident branch has a countable carrier.

Proof Let $E$ be a complete evident branch. We define:

$$
\begin{aligned}
D & :=\left\{\tilde{s} \mid s \in \mathrm{EFO}_{\iota}\right\} \\
s \triangleright_{\iota} \tilde{t} & : \Leftrightarrow s \sim t
\end{aligned}
$$

We will show that $\left(D, \triangleright_{l}\right)$ is a carrier for $E$. Note that $\triangleright_{l}$ is well-defined since is an equivalence relation. $D$ is countable since $\mathrm{EFO}_{\iota}$ is countable.

B1. We have to show that $s \sim t$ iff $[s] \sim t$. This follows with N3 and N1 since $s \sim t$ iff $[s=t] \in E$ and $[s] \sim t$ iff $[[s]=t] \in E$.

B2. If $T$ is empty, B2 holds vacuously. Otherwise, let $t \in T$. Then $T$ is compatible iff $s \sim t$ for all $s \in T$ by Propositions 6.3 and 6.2. Hence $T$ is compatible iff $s \triangleright_{\text {, }} t$ for all $s \in T$. The claim follows.

B3. Let $s={ }_{\iota} t$ in $E$ and $s \triangleright_{\iota} \tilde{u}$ and $t \nabla_{\iota} \tilde{v}$. Since $s=t$ is normal, we have $s \sim t$. By definition of $\triangleright_{\text {, we have }} \sim u$ and $t \sim v$. Hence $\tilde{u}=\tilde{v}$ since $\sim$ is an equivalence relation.

B4. If $\mathcal{D E}$ is empty, then $s \triangleright_{\iota} \tilde{t}$ for all $s, t \in \mathrm{EFO}_{\iota}$ and hence the claim holds. Otherwise, let $\mathcal{D E}$ be nonempty. We show the claim by contradiction. Suppose there is a term $t \in \mathrm{EFO}_{\iota}$ such that $s \not \iota \tilde{t}$ for all $s \in \mathcal{D} E$. Then $[s \neq t] \in E$ for all $s \in \mathcal{D} E$ by Proposition 6.1. Since $\mathcal{D} E$ is nonempty, we have $[t] \in \mathcal{D} E$ by N3. Thus $([t] \neq[t]) \in E$ by N3. Contradiction by $\mathcal{E}_{\neq}$.

\subsection{Discriminant-Based Carriers}

We will now show that every evident branch has a carrier. Let an evident branch $E$ be given. We will call a term discriminating if it is discriminating in $E$. A discriminant is a maximal set $a$ of discriminating terms such that there is no disequation $s \neq t \in E$ such that $s, t \in a$. We will construct a carrier for $E$ whose values are the discriminants.

Example 6.5 Suppose $E=\{x \neq y, x \neq z, y \neq z\}$ and $x, y, z: \iota$. Then there are 3 discriminants: $\{x\},\{y\},\{z\}$.

Example 6.6 Suppose $E=\{x \neq f(f x), f x \neq f(f(f x))\}$ and $f: \iota$. Then there are 4 discriminants: $\{x, f x\},\{x, f(f(f x))\},\{f(f x), f x\},\{f(f x), f(f(f x))\}$ • 
Example 6.7 Suppose $E=\left\{a_{n} \neq{ }_{\iota} b_{n} \mid n \in \mathbb{N}\right\}$ where the $a_{n}$ and $b_{n}$ are pairwise distinct constants. Then $E$ is evident and there are uncountably many discriminants.

Proposition 6.8 If $E$ contains exactly $n$ disequations at $\iota$, then there are at most $2^{n}$ discriminants. If $E$ contains no disequation at $\iota$, then $\emptyset$ is the only discriminant.

Proposition 6.9 Let $a$ and $b$ be different discriminants. Then:

1. $a$ and $b$ are separated by a disequation in $E$, that is, there exist terms $s \in a$ and $t \in b$ such that $s \sharp t$.

2. $a$ and $b$ are not connected by an equation in $E$, that is, there exist no terms $s \in a$ and $t \in b$ such that $(s=t) \in E$.

Proof The first claim follows by contradiction. Suppose there are no terms $s \in a$ and $t \in b$ such that $s \sharp t$. Let $s \in a$. Then $s \in b$ since $b$ is a maximal compatible set of discriminating terms. Thus $a \subseteq b$ and hence $a=b$ since $a$ is maximal. Contradiction.

The second claim also follows by contradiction. Suppose there is an equation $\left(s_{1}=s_{2}\right) \in E$ such that $s_{1} \in a$ and $s_{2} \in b$. By the first claim we have terms $s \in a$ and $t \in b$ such that $s \sharp t$. By $\mathcal{E}_{\mathrm{CON}}$ we have $s_{1} \sharp s$ or $s_{2} \sharp t$. Contradiction since $a$ and $b$ are discriminants.

Lemma 6.10 Every (finite) evident branch has a (finite) carrier.

Proof Let $E$ be an evident branch. We define:

$$
\begin{aligned}
D & :=\text { set of all discriminants } \\
s \triangleright_{\iota} a & : \Leftrightarrow([s] \text { discriminating } \Longrightarrow[s] \in a)
\end{aligned}
$$

We will show that $\left(D, \triangleright_{\iota}\right)$ is a carrier for $E$. By Proposition 6.8 we know that $D$ is finite if $E$ is finite.

B1. Holds by N1.

For the remaining carrier conditions we distinguish two cases. If $\mathcal{D} E=\emptyset$, then $\emptyset$ is the only discriminant and B2, B3, and B4 are easily verified. Otherwise, let $\mathcal{D} E \neq \emptyset$.

B2 $\Rightarrow$. Let $T$ be compatible. Then there exists a discriminant $a$ that contains all the discriminating terms in $\{[t] \mid t \in T\}$. The claim follows since $T \triangleright a$.

B2 $\Leftarrow$. By contradiction. Suppose $T \triangleright a$ and $T$ is not compatible. Then there are terms $s, t \in T$ such that $([s] \neq[t]) \in E$. Thus $[s]$ and $[t]$ cannot be both in $a$. This contradicts $s, t \in T \triangleright a$ since $[s]$ and $[t]$ are discriminating. 
B3. Let $(s=t) \in E$ and $s \triangleright_{\imath} a$ and $t \triangleright_{\imath} b$. We show $a=b$. Since there are discriminating terms, $E$ contains at least one disequation at type $\iota$, and hence $s$ and $t$ are discriminating by $\mathcal{E}_{\mathrm{CON}}$. By N3 $s$ and $t$ are normal and hence $s \in a$ and $t \in b$. Now $a=b$ by Proposition 6.9(2).

B4. Since there are discriminating terms, we know by $\mathcal{E}_{\neq}$that every discriminant contains at least one discriminating term. Since discriminating terms are normal, we have the claim.

\section{Model Existence}

We will now show that every evident branch has a model.

Lemma 7.1 (Model Existence) Let $\left(D, \triangleright_{\iota}\right)$ be a carrier for an evident branch $E$. Then $E$ has a model $\mathcal{I}$ such that $\mathcal{I} \iota=D$.

We start the proof of Lemma 7.1 Let $\left(D, \triangleright_{\iota}\right)$ be a carrier for an evident branch $E$. For the rest of the proof we only consider interpretations $\mathcal{I}$ such that $\mathcal{I} \iota=D$.

\subsection{Possible Values}

To obtain a model of $E$, we need suitable values for all variables. We address this problem by defining possible-values relations $\triangleright_{\sigma} \subseteq \mathrm{EFO}_{\sigma} \times \mathcal{I} \sigma$ for all types $\sigma \neq \imath$ :

$$
\begin{aligned}
s \triangleright_{o} 0 & : \Leftrightarrow[s] \notin E \\
s \triangleright_{o} 1 & : \Leftrightarrow \neg[s] \notin E \\
s \triangleright_{\sigma \tau} f & : \Leftrightarrow s t \triangleright_{\tau} f a \text { whenever } t \triangleright_{\sigma} a
\end{aligned}
$$

Note that we already have a possible-values relation for $\iota$ and that the definition of the possible values relations for functional types is by induction on types. Also note that if $s$ is an EFO formula such that $[s] \notin E$ and $\neg[s] \notin E$, then both 0 and 1 are possible values for $s$. We will show that every EFO term has a possible value and that we obtain a model of $E$ if we define $\mathcal{I} x$ as a possible value for $x$ for every variable $x$.

Proposition 7.2 Let $s \in \mathrm{EFO}_{\sigma}$ and $a \in \mathcal{I} \sigma$. Then $s \triangleright_{\sigma} a \Longleftrightarrow[s] \triangleright_{\sigma} a$.

Proof By induction on $\sigma$. For $o$ the claim follows with N1. For $\iota$ the claim follows with B1. Let $\sigma=\tau \mu$.

Suppose $s \triangleright_{\sigma} a$. Let $t \triangleright_{\tau} b$. Then $s t \triangleright_{\mu} a b$. By inductive hypothesis $\left[s t \triangleright_{\mu} a b\right.$. Thus $[[s] t] \triangleright_{\mu} a b$ by N2. By inductive hypothesis $[s] t \triangleright_{\mu} a b$. Hence $[s] \triangleright_{\sigma} a$. 
Suppose $[s] \triangleright_{\sigma} a$. Let $t \triangleright_{\tau} b$. Then $[s] t \triangleright_{\mu} a b$. By inductive hypothesis $[[s] t] \triangleright_{\mu} a b$. Thus $[s t] \triangleright_{\mu} a b$ by N2. By inductive hypothesis $s t \triangleright_{\mu} a b$. Hence $s \triangleright_{\sigma} a$.

Lemma 7.3 For every EFO constant $c: c \triangleright \mathcal{I} c$.

Proof

$c=\perp$. The claim follows by $\mathcal{E}_{\perp}$ and N3.

$c=\neg$. Assume $s \triangleright_{o} a$. We show $\neg \mathcal{S} \triangleright \mathcal{I}(\neg) a$ by contradiction. Suppose $\neg \mathcal{S} \not$ $\mathcal{I}(\neg) a$. Case analysis.

$a=0$. Then $[s] \notin E$ and $\neg[\neg s] \in E$. Thus $\neg \neg[s] \in E$ by N3. Hence $[s] \in E$ by

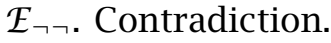

$a=1$. Then $\neg[s] \notin E$ and $[\neg s] \in E$. Contradiction by N3.

$c=\wedge$. Assume $s \triangleright_{o} a$ and $t \triangleright_{o} b$. We show $s \wedge t \triangleright \mathcal{I}(\wedge) a b$ by contradiction. Suppose $s \wedge t \not \mathcal{I}(\wedge) a b$. Case analysis.

$a=b=1$. Then $\neg[s], \neg[t] \notin E$ and $\neg[s \wedge t] \in E$. Contradiction by N3 and $\mathcal{E}_{\neg \wedge}$.

$a=0$ or $b=0$. Then $[s] \notin E$ or $[t] \notin E$, and $[s \wedge t] \in E$. Contradiction by N3 and $\mathcal{E}_{\wedge}$.

$c=(=\imath)$. Assume $s \triangleright_{\imath} a$ and $t \triangleright_{\iota} b$. We show $(s=t) \triangleright \mathcal{I}(=) a$,$b by contradiction.$ Suppose $(s=t) \not \mathcal{I}(=\iota) a b$. Case analysis.

$a=b$. Then $\neg[s=t] \in E$ and $s, t \triangleright, a$. By B2 $\{s, t\}$ is compatible. Contradiction by N3.

$a \neq b$. Then $([s]=[t]) \in E$ by N3. Hence $a=b$ by B1 and B3. Contradiction. $c=\forall_{\iota}$. Assume $s \triangleright_{\iota o} f$. We show $\forall_{\iota} s \triangleright_{o} \mathcal{I} \forall_{\iota} f$ by contradiction. Suppose $\forall_{\iota} s \not_{o}$ $\mathcal{I} \forall, f$. Case analysis.

$\mathcal{I} \forall_{\iota} f=0$. Then $\forall_{\iota}[s] \in E$ by N3 and $f a=0$ for some value $a$. By $\mathcal{E}_{\forall}$ and B4 there exists a term $t$ such that $[[s] t] \in E$ and $t \triangleright_{\imath} a$. Thus st $\triangleright f a=0$ and hence $[s t] \notin E$. Contradiction by N2.

$\mathcal{I} \forall_{\iota} f=1$. Then $\neg \forall_{\iota}[s] \in E$ by N3. By $\mathcal{E}_{\neg \forall}$ we have $\neg[[s] t] \in E$ for some term $t \in \mathrm{EFO}_{\iota}$. By $\mathcal{E}_{\neq}$and B2 we have $t \triangleright a$ for some value $a$. Now st $\triangleright f a=1$. Thus $\neg[s t] \notin E$. Contradiction by N2.

We call an interpretation $\mathcal{I}$ admissible if it satisfies $x \triangleright \mathcal{I} x$ for every variable $x$. We will show that admissible interpretations exist and that every admissible interpretation is a model of $E$.

Lemma 7.4 (Admissibility) Let $\mathcal{I}$ be admissible and $\theta$ be a substitution such that $\theta x \triangleright \mathcal{I} x$ for all $x \in \operatorname{Dom} \theta$. Then $\hat{\theta} s \triangleright \hat{\mathcal{I}} s$ for every EFO term $s$. 
Proof By induction on $s$. Let $s$ be an EFO term. By assumption, $\theta x$ is EFO for all $x \in \operatorname{Dom} \theta$. Hence $\hat{\theta} s$ is EFO by S5. Case analysis.

$s=a$. If $a \in \operatorname{Dom} \theta$, the claim holds by assumption. If $a \notin \operatorname{Dom} \theta$, then $\hat{\theta} s=a$ by S1. If $a$ is a constant, the claim holds by Lemma 7.3. If $a$ is a variable, the claim holds by assumption.

$s=t u$. Then $\hat{\theta} s=(\hat{\theta} t)(\hat{\theta} u)$ by S2. Now $\hat{\theta} t \triangleright \hat{I} t$ and $\hat{\theta} u \triangleright \hat{\mathcal{I}} u$ by the inductive hypothesis. Now $\hat{\theta} s=(\hat{\theta} t)(\hat{\theta} u) \triangleright(\hat{\mathfrak{I}} t)(\hat{\mathfrak{I}} u)=\hat{\mathfrak{I}} s$.

$s=\lambda x$.t and $x: \sigma$. Moreover, let $u \triangleright_{\sigma} a$. We show $(\hat{\theta} s) u \triangleright(\hat{I} s) a$. By Proposition 7.2 it suffices to show $[(\hat{\theta} s) u] \triangleright(\hat{\mathcal{I}} s) a$. We have $[(\hat{\theta} s) u]=\left[\widehat{\theta_{u}^{x}} t\right]$ by S3 and $(\hat{\mathcal{I}} s) a=\widehat{\mathcal{I}}_{a}^{x} t$ where $\mathcal{I}_{a}^{x}$ denotes the interpretation that agrees everywhere with $\mathcal{I}$ but possibly on $x$ where it yields $a$. By inductive hypothesis we have $\widehat{\theta_{u}^{x}} t \triangleright \widehat{\mathcal{I}_{a}^{x}} t$. The claim follows with Proposition 7.2 .

\subsection{Compatibility}

It remains to show that there is an admissible interpretation and that every admissible interpretation is a model of $E$. For this purpose we define compatibility relations $\|_{\sigma} \subseteq \mathrm{EFO}_{\sigma} \times \mathrm{EFO}_{\sigma}$ for all types:

$$
\begin{aligned}
s \|_{o} t & : \Longleftrightarrow\{[s], \neg[t]\} \nsubseteq E \text { and }\{\neg[s],[t]\} \nsubseteq E \\
s \|_{\iota} t & : \Longleftrightarrow \operatorname{not}[s] \#[t] \\
s \|_{\sigma \tau} t & : \Longleftrightarrow s u \|_{\tau} t v \text { whenever } u \|_{\sigma} v
\end{aligned}
$$

Note that the definition of the compatibility relations for functional types is by induction on types. We say that $s$ and $t$ are compatible if $s \| t$. A set $T$ of equityped terms is compatible if $s \| t$ for all terms $s, t \in T$. If $T \subseteq \mathrm{EFO}_{\sigma}$, we write $T \triangleright a$ if $a$ is a common possible value for all terms $s \in T$. We will show that a set of equi-typed terms is compatible if and only if all its terms have a common possible value.

Proposition 7.5 The compatibility relations $\|_{\sigma}$ are symmetric.

The compatibility relations are also reflexive. Showing this fact will take some effort. We first show $x \| x$ for all variables $x$. For the induction to go through we strengthen the hypothesis.

Lemma 7.6 (Reflexivity) For every type $\sigma$ and all EFO terms $s, t, x s_{1} \ldots s_{n}$, $x t_{1} \ldots t_{n}$ of type $\sigma$ with $n \geq 0$ :

1. Not both $s \|_{\sigma} t$ and $[s] \sharp[t]$.

2. Either $x s_{1} \ldots s_{n} \|_{\sigma} x t_{1} \ldots t_{n}$ or $\left[s_{i}\right] \#\left[t_{i}\right]$ for some $i \in\{1, \ldots, n\}$. 
Proof By mutual induction on $\sigma$. The base cases for Claim (1) follow by contradiction. For $\sigma=o \mathcal{E}_{\mathrm{BE}}$ is needed. The base cases for Claim (2) follow with N3, $\mathcal{E}_{\mathrm{MAT}}, \mathcal{E}_{\mathrm{DEC}}, \mathcal{E}_{\neg}$, and $\mathcal{E}_{\neq}$. We now show the claims for $\sigma=\tau \mu$.

1. By contradiction. Suppose $s \|_{\sigma} t$ and $[s] \#[t]$. By $\mathcal{E}_{\mathrm{FE}}[[s] x] \#[[t] x]$ for some variable $x$. By inductive hypothesis (2) we have $x \|_{\tau} x$. Hence $s x \|_{\mu} t x$. Contradiction by inductive hypothesis (1) and N2.

2. Suppose $x s_{1} \ldots s_{n} \sharp_{\sigma} x t_{1} \ldots t_{n}$. Then there exist terms such that $u \|_{\tau} v$ and $x s_{1} \ldots s_{n} u \nVdash \mu x t_{1} \ldots t_{n} v$. By inductive hypothesis (1) we know that $[u] \#[v]$ does not hold. Hence $\left[s_{i}\right] \#\left[t_{i}\right]$ for some $i \in\{1, \ldots, n\}$ by inductive hypotheses (2).

Lemma 7.7 (Common Value) Let $T \subseteq \mathrm{EFO}_{\sigma}$. Then $T$ is compatible if and only if there exists a value $a$ such that $T \triangleright_{\sigma} a$.

Proof By induction on $\sigma$. For $\sigma=\iota$ the claim is identical with B2.

$\sigma=o, \Rightarrow$. By contraposition. Suppose $T \not 0$ and $T \not 1$. Then there are terms $s, t \in T$ such that $[s], \neg[t] \in E$. Thus $s \nVdash t$. Hence $T$ is not compatible.

$\sigma=o, \Leftarrow$. By contraposition. Suppose $s \sharp_{o} t$ for $s, t \in T$. Then $[s], \neg[t] \in E$ without loss of generality. Hence $s \not 0$ and $t \not 1$. Thus $T \not 0$ and $T \not 1$.

$\sigma=\tau \mu, \Rightarrow$. Let $T$ be compatible. We define $T_{a}:=\left\{t s \mid t \in T, s \triangleright_{\tau} a\right\}$ for every value $a \in \mathcal{I} \tau$ and show that $T_{a}$ is compatible. Let $t_{1}, t_{2} \in T$ and $s_{1}, s_{2} \triangleright_{T} a$. It suffices to show $t_{1} s_{1} \| t_{2} s_{2}$. By the inductive hypothesis $s_{1} \|_{T} s_{2}$. Since $T$ is compatible, $t_{1} \| t_{2}$. Hence $t_{1} s_{1} \| t_{2} s_{2}$.

By the inductive hypothesis we now know that for every $a \in \mathcal{I} \tau$ there is a $b \in \mathcal{I} \mu$ such that $T_{a} \triangleright_{\mu} b$. Hence there is a function $f \in \mathcal{I} \sigma$ such that $T_{a} \triangleright_{\mu} f a$ for every $a \in \mathcal{I} \tau$. Thus $T \triangleright_{\sigma} f$.

$\sigma=\tau \mu, \Leftarrow$. Let $T \triangleright_{\sigma} f$ and $s, t \in T$. We show $s \|_{\sigma} t$. Let $u \|_{\tau} v$. It suffices to show $s u \|_{\mu} t v$. By the inductive hypothesis $u, v \triangleright_{T} a$ for some value $a$. Hence $s u, t v \triangleright_{\mu} f a$. Thus $s u \|_{\mu} t v$ by the inductive hypothesis.

Lemma 7.8 Every admissible interpretation is a model of $E$.

Proof Let $\mathcal{I}$ be an admissible interpretation and $s \in E$. We show $\hat{\mathcal{I}} s=1$. Case analysis.

Suppose $s$ is a normal EFO term. Then $s=[s]=[\hat{\emptyset} s]$ by S4 and $s \not 0$. Moreover, $\hat{\emptyset} s \hat{\mathcal{I}} s$ by Lemma 7.4 and $s \triangleright \hat{\mathcal{I}} s$ by Lemma 7.2. Hence $\hat{\mathcal{I}} s=1$.

Suppose $s=(t \neq u)$ where $t$ and $u$ are normal EFO terms. Then $t=[t]=[\hat{\emptyset} t]$ and $u=[u]=[\hat{\emptyset} u]$ by S4. We prove the claim by contradiction. Suppose $\hat{\mathcal{I}} s=0$. Then $\hat{\mathcal{I}} t=\hat{\mathfrak{I}} u$. Thus $\hat{\emptyset} t, \hat{\emptyset} u \triangleright \hat{\mathcal{I}} t$ by Lemma 7.4 and $t, u \triangleright \hat{\mathcal{I}} t$ by Lemma 7.2. Hence 
$t \| u$ by Lemma 7.7 Thus not $[t] \#[u]$ by Lemma [7.6](1). Contradiction since $([t] \neq[u]) \in E$.

We can now prove Lemma 7.1. By Lemma 7.6(2) we know $x \| x$ for every variable $x$. Hence there exists an admissible interpretation $\mathcal{I}$ by Lemma 7.7. By Lemma 7.8 we know that $\mathcal{I}$ is a model of $E$. This finishes the proof of Lemma 7.1 .

Theorem 7.9 (Finite Model Existence)

Every finite evident branch has a finite model.

Proof Follows with Lemmas 6.10 and 7.1.

Lemma 7.10 (Model Existence) Let $E$ be an evident branch. Then $E$ has a model. Moreover, $E$ has a countable model if $E$ is complete.

Proof Follows with Lemmas 6.10, 7.1, and 6.4

\section{Abstract Consistency}

To obtain our main results, we boost the model existence lemma with the abstract consistency technique. Everything works out smoothly.

An abstract consistency class is a set $\Gamma$ of branches such that every branch $A \in \Gamma$ satisfies the conditions in Figure 3. An abstract consistency class $\Gamma$ is complete if for every $A \in \Gamma$ and all $s, t \in \mathrm{EFO}_{\iota}$ either $A \cup\{[s=t]\}$ is in $\Gamma$ or $A \cup\{[s \neq t]\}$ is in $\Gamma$.

Proposition 8.1 Let $A$ be a branch. Then $A$ is evident if and only if $\{A\}$ is an abstract consistency class. Moreover, $A$ is a complete evident branch if and only if $\{A\}$ is a complete abstract consistency class.

Lemma 8.2 (Extension Lemma) Let $\Gamma$ be an abstract consistency class and $A \in \Gamma$. Then there exists an evident branch $E$ such that $A \subseteq E$. Moreover, if $\Gamma$ is complete, a complete evident branch $E$ exists such that $A \subseteq E$.

Proof Let $u_{0}, u_{1}, u_{2}, \ldots$ be an enumeration of all formulas that can occur on a branch. We construct a sequence $A_{0} \subseteq A_{1} \subseteq A_{2} \subseteq \cdots$ of branches such that every $A_{n} \in \Gamma$. Let $A_{0}:=A$. We define $A_{n+1}$ by cases. If there is no $B \in \Gamma$ such that $A_{n} \cup\left\{u_{n}\right\} \subseteq B$, then let $A_{n+1}:=A_{n}$. Otherwise, choose some $B \in \Gamma$ such that $A_{n} \cup\left\{u_{n}\right\} \subseteq B$. We consider four subcases.

1. If $u_{n}$ is of the form $\forall_{l} s$, then choose $A_{n+1}$ to be $B \cup\{[s t]\} \in \Gamma$ for some $t \in \mathrm{EFO}_{\iota}$. This is possible since $\Gamma$ satisfies $C_{\forall}$. 


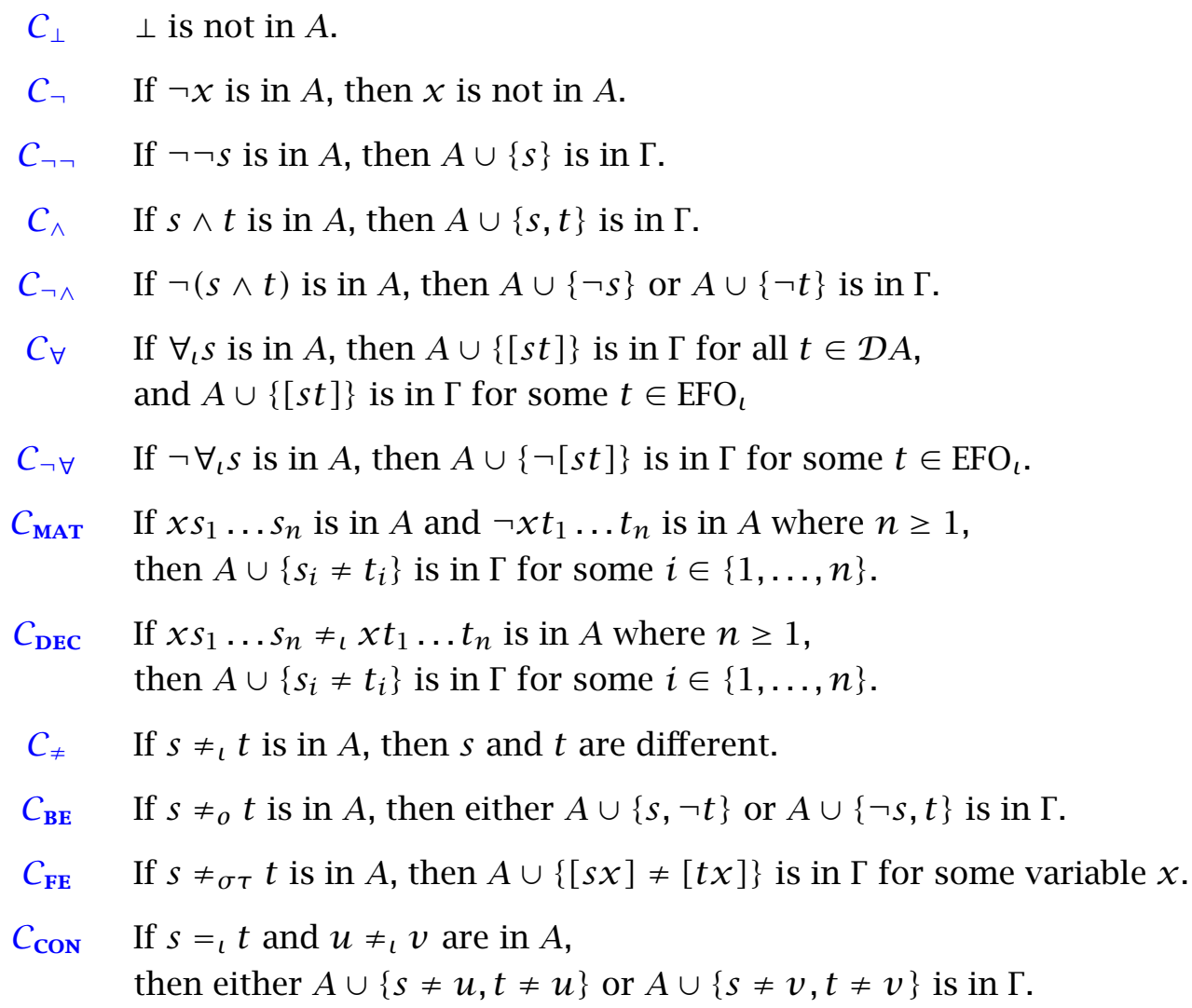

Figure 3: Abstract consistency conditions (must hold for every $A \in \Gamma$ )

2. If $u_{n}$ is of the form $\neg \forall_{l} s$, then choose $A_{n+1}$ to be $B \cup\{\neg[s t]\} \in \Gamma$ for some $t \in \mathrm{EFO}_{\iota}$. This is possible since $\Gamma$ satisfies $C_{\neg \forall}$.

3. If $u_{n}$ is of the form $s \neq{ }_{\sigma \tau} t$, then choose $A_{n+1}$ to be $B \cup\{[s x] \neq[t x]\} \in \Gamma$ for some variable $x$. This is possible since $\Gamma$ satisfies $C_{\mathrm{FE}}$.

4. If $u_{n}$ has none of these forms, then let $A_{n+1}$ be $B$.

Let $E:=\bigcup_{n \in \mathbb{N}} A_{n}$. Note that $\mathcal{D} E=\bigcup_{n \in \mathbb{N}} \mathcal{D} A_{n}$. We show that $E$ is evident.

$\mathcal{E}_{\perp}$ If $\perp$ is in $E$, then $\perp$ is in $A_{n}$ for some $n$, contradicting $C_{\perp}$.

$\mathcal{E}_{\neg} \quad$ If $\neg x$ and $x$ are in $E$, then $\neg x$ and $x$ are in $A_{n}$ for some $n$, contradicting $C_{\neg}$.

$\mathcal{E}_{\neq}$If $s \neq{ }_{\iota} s$ is in $E$, then $s \neq{ }_{\iota} s$ is in $A_{n}$ for some $n$, contradicting $C_{\neq}$.

$\mathcal{E}_{\neg \neg}$ Assume $\neg \neg s$ is in $E$. Let $n$ be such that $u_{n}=s$ and $r \geq n$ be such that $\neg \neg s$ is in $A_{r}$. Since $A_{n} \cup\{s\} \subseteq A_{r} \cup\{s\} \in \Gamma$ (using $C_{\neg \neg}$ ), we have $s \in A_{n+1} \subseteq E$.

$\mathcal{E}_{\wedge}$ Assume $s \wedge t$ is in $E$. Let $n, m$ be such that $u_{n}=s$ and $u_{m}=t$. Let $r \geq n, m$ be such that $s \wedge t$ is in $A_{r}$. By $C_{\wedge}, A_{r} \cup\{s, t\} \in \Gamma$. Since $A_{n} \cup\{s\} \subseteq A_{r} \cup\{s, t\}$, 
we have $s \in A_{n+1} \subseteq E$. Since $A_{m} \cup\{t\} \subseteq A_{r} \cup\{s, t\}$, we have $t \in A_{m+1} \subseteq E$.

$\mathcal{E}_{\neg \wedge}$ Assume $\neg(s \wedge t)$ is in $E$. Let $n, m$ be such that $u_{n}=s$ and $u_{m}=t$. Let $r \geq n, m$ be such that $\neg(s \wedge t)$ is in $A_{r}$. By $C_{\neg \wedge}, A_{r} \cup\{\neg s\} \in \Gamma$ or $A_{r} \cup\{\neg t\} \in \Gamma$. In the first case, $A_{n} \cup\{\neg s\} \subseteq A_{r} \cup\{\neg s\} \in \Gamma$, and so $\neg s \in A_{n+1} \subseteq E$. In the second case, $A_{m} \cup\{\neg t\} \subseteq A_{r} \cup\{\neg t\} \in \Gamma$, and so $\neg t \in A_{m+1} \subseteq E$. Hence either $\neg S$ or $\neg t$ is in $E$.

$\mathcal{E}_{\forall} \quad$ Assume $\forall_{l} s$ is in $E$. Case analysis.

$\mathcal{D} E=\emptyset$. Let $n$ be such that $u_{n}=\forall_{l} s$. Let $r \geq n$ be such that $\forall_{l} s$ is in $A_{r}$.

By $C_{\forall}$ there is some $t$ such that $A_{r} \cup\{[s t]\}$ is in $\Gamma$. Hence $[s t] \in A_{n+1} \subseteq E$ for some $t$.

$\mathcal{D} E \neq \emptyset$. Let $t \in \mathcal{D} E$. We show $[s t] \in E$. Let $n$ be such that $u_{n}=[s t]$. Let

$r \geq n$ be such that $\forall_{l} s$ is in $A_{r}$ and $t \in \mathcal{D} A_{r}$. By $C_{\forall}$ we have $A_{r} \cup\{[s t]\} \in \Gamma$.

Since $A_{n} \cup\left\{u_{n}\right\} \subseteq A_{r} \cup\{[s t]\}$, we have $[s t]=u_{n} \in A_{n+1} \subseteq E$.

$\mathcal{E}_{\neg \forall} \quad$ Assume $\neg \forall_{l} s$ is in $E$. Let $n$ be such that $u_{n}=\neg \forall_{l} s$. Let $r \geq n$ be such that $\neg \forall_{l} s$ is in $A_{r}$. By $C_{\neg \forall}$ we know $A_{r} \cup\{\neg[s t]\} \in \Gamma$ for some $t$. Hence $\neg[s t] \in A_{n+1} \subseteq E$ for some $t$.

$\mathcal{E}_{\mathrm{MAT}}$ Assume $x s_{1} \ldots s_{n}$ and $\neg x t_{1} \ldots t_{n}$ are in $E$ for some $n \geq 1$. For each $i \in$ $\{1, \ldots, n\}$, let $m_{i}$ be such that $u_{m_{i}}$ is $s_{i} \neq t_{i}$. Let $r \geq m_{1}, \ldots, m_{n}$ be such that $x s_{1} \ldots s_{n}$ and $\neg x t_{1} \ldots t_{n}$ are in $A_{r}$. By $C_{\mathrm{MAT}}$ there is some $i \in\{1, \ldots, n\}$ such that $A_{r} \cup\left\{s_{i} \neq t_{i}\right\} \in \Gamma$. Since $A_{n} \cup\left\{s_{i} \neq t_{i}\right\} \subseteq A_{r} \cup\left\{s_{i} \neq t_{i}\right\}$, we have $\left(s_{i} \neq t_{i}\right) \in A_{n+1} \subseteq E$.

$\mathcal{E}_{\mathrm{DEC}}$ Assume $x s_{1} \ldots s_{n} \neq_{\iota} x t_{1} \ldots t_{n}$ is in $E$ for some $n \geq 1$. For each $i \in$ $\{1, \ldots, n\}$, let $m_{i}$ be such that $u_{m_{i}}$ is $s_{i} \neq t_{i}$. Let $r \geq m_{1}, \ldots, m_{n}$ be such that $x s_{1} \ldots s_{n} \neq \imath x t_{1} \ldots t_{n}$ is in $A_{r}$. By $C_{\mathrm{DEC}}$ there is some $i \in\{1, \ldots, n\}$ such that $A_{r} \cup\left\{s_{i} \neq t_{i}\right\} \in \Gamma$. Since $A_{n} \cup\left\{s_{i} \neq t_{i}\right\} \subseteq A_{r} \cup\left\{s_{i} \neq t_{i}\right\}$, we have $\left(s_{i} \neq t_{i}\right) \in A_{n+1} \subseteq E$.

$\mathcal{E}_{\mathrm{BE}}$ Assume $s \neq_{o} t$ is in $E$. Let $n, m, j, k$ be such that $u_{n}=s, u_{m}=t, u_{j}=\neg s$ and $u_{k}=\neg t$. Let $r \geq n, m, j, k$ be such that $s \neq_{o} t$ is in $A_{r}$. By $C_{\mathrm{BE}}$ either $A_{r} \cup\{s, \neg t\}$ or $A_{r} \cup\{\neg s, t\}$ is in $\Gamma$. Assume $A_{r} \cup\{s, \neg t\}$ is in $\Gamma$. Since $A_{n} \cup\{s\} \subseteq$ $A_{r} \cup\{s, \neg t\}$, we have $s \in E$. Since $A_{k} \cup\{\neg t\} \subseteq A_{r} \cup\{s, \neg t\}$, we have $\neg t \in E$. Next assume $A_{r} \cup\{\neg s, t\}$ is in $\Gamma$. Since $A_{j} \cup\{\neg S\} \subseteq A_{r} \cup\{\neg S, t\}$, we have $\neg s \in E$. Since $A_{m} \cup\{t\} \subseteq A_{r} \cup\{\neg s, t\}$, we have $t \in E$.

$\mathcal{E}_{\mathrm{FE}}$ Assume $s \neq_{\sigma \tau} t$ is in $E$. Let $n$ be such that $u_{n}$ is $s \neq_{\sigma \tau} t$. Let $r \geq n$ be such that $s \neq_{\sigma \tau} t$ is in $A_{r}$. Since $A_{n} \cup\left\{u_{n}\right\} \subseteq A_{r}$, there is some variable $x$ such that $[s x] \neq_{\tau}[t x]$ is in $A_{n+1} \subseteq E$.

$\mathcal{E}_{\text {Con }}$ Assume $s={ }_{\iota} t$ and $u \neq \imath v$ are in $E$. Let $n, m, j, k$ be such that $u_{n}$ is $s \neq u, u_{m}$ is $t \neq u, u_{j}$ is $s \neq v$ and $u_{k}$ is $t \neq v$. Let $r \geq n, m, j, k$ be such that $s={ }_{\iota} t$ and $u \neq \imath, v$ are in $A_{r}$. By $C_{\mathrm{CON}}$ either $A_{r} \cup\{s \neq u, t \neq u\}$ 
or $A_{r} \cup\{s \neq v, t \neq v\}$ is in $\Gamma$. Assume $A_{r} \cup\{s \neq u, t \neq u\}$ is in $\Gamma$. Since $A_{n} \cup\{s \neq u\} \subseteq A_{r} \cup\{s \neq u, t \neq u\}$, we have $s \neq u \in A_{n+1} \subseteq E$. Since $A_{m} \cup\{t \neq u\} \subseteq A_{r} \cup\{s \neq u, t \neq u\}$, we have $t \neq u \in A_{m+1} \subseteq E$. Next assume $A_{r} \cup\{s \neq v, t \neq v\}$ is in $\Gamma$. Since $A_{j} \cup\{s \neq v\} \subseteq A_{r} \cup\{s \neq v, t \neq v\}$, we have $s \neq v \in A_{j+1} \subseteq E$. Since $A_{k} \cup\{t \neq v\} \subseteq A_{r} \cup\{s \neq v, t \neq v\}$, we have $t \neq v \in A_{k+1} \subseteq E$.

It remains to show that $E$ is complete if $\Gamma$ is complete. Let $\Gamma$ be complete and $s, t \in \mathrm{EFO}_{\iota}$. We show that $[s=t]$ or $[s \neq t]$ is in $E$. Let $m, n$ be such that $u_{m}=[s=t]$ and $u_{n}=[s \neq t]$. We consider $m<n$, the case $m>n$ is symmetric. If $[s=t] \in A_{n}$, we have $[s=t] \in E$. If $[s=t] \notin A_{n}$, then $A_{n} \cup\{[s=t]\}$ is not in $\Gamma$. Hence $A_{n} \cup\{[s \neq t]\}$ is in $\Gamma$ since $\Gamma$ is complete. Hence $[s \neq t] \in A_{n+1} \subseteq E$.

\section{Completeness}

We will now show that the tableau system $\mathcal{T}$ is complete. In fact, we will show the completeness of a tableau system $\mathcal{R}$ that is obtained from $\mathcal{T}$ by restricting the applicability of some of the rules. We consider $\mathcal{R}$ since it provides for more focused proof search and also yields a decision procedure for three substantial fragments of EFO. $\mathcal{R}$ is obtained from $\mathcal{T}$ by restricting the applicability of the rules $\mathcal{T}_{\forall}, \mathcal{T}_{\neg \forall}$, and $\mathcal{T}_{\mathrm{FE}}$ as follows:

- $\mathcal{T}_{\forall}$ can only be applied to $\forall_{\iota} s \in A$ with a term $t \in \mathrm{EFO}_{\iota}$ if either $t \in \mathcal{D} A$ or the following conditions are satisfied:

1. $\mathcal{D} A=\emptyset$ and $t$ is a variable.

2. $t \in \mathcal{N} A$ or $\mathcal{N} A=\emptyset$.

3. There is no $u \in \mathrm{EFO}_{\iota}$ such that $[s u] \in A$.

- $\mathcal{T}_{\neg \forall}$ can only be applied to $\neg \forall_{\iota} s \in A$ if there is no $t \in \mathrm{EFO}_{\iota}$ such that $\neg[s t] \in A$.

- $\mathcal{T}_{\mathrm{FE}}$ can only be applied to an equation $\left(s={ }_{\sigma \tau} t\right) \in A$ if there is no variable $x: \sigma$ such that $([s x]=[t x]) \in A$.

We use $\mathcal{R}_{\forall}, \mathcal{R}_{\neg \forall}$, and $\mathcal{R}_{\mathrm{FE}}$ to refer to the restrictions of $\mathcal{T}_{\forall}, \mathcal{T}_{\neg \forall}$, and $\mathcal{T}_{\mathrm{FE}}$, respectively. Note that $\mathcal{R}_{\forall}$ provides a novel subterm restriction that may be useful for proof search. We say a branch $A$ is refutable if it can be refuted with $\mathcal{R}$. Let $\Gamma_{\mathcal{T}}$ be the set of all finite branches that are not refutable.

Lemma 9.1 $\Gamma_{\mathcal{T}}$ is an abstract consistency class.

Proof We have to show that $\Gamma_{\mathcal{T}}$ satisfies the abstract consistency conditions. We prove some of the conditions, the verification of the remaining conditions is straightforward. 
$C_{\perp}$ Suppose $\perp \in A \in \Gamma_{\mathcal{T}}$. Then $A$ is refutable. Contradiction.

$C_{\neg}$ Suppose $\neg x, x \in A \in \Gamma_{\mathcal{T}}$. Then we can refute $A$ using $\mathcal{T}_{\neg}$. Contradiction.

$C_{\neg \wedge}$ Let $\neg(s \wedge t) \in A \in \Gamma_{\mathcal{T}}$. Suppose $A \cup\{\neg s\}$ and $A \cup\{\neg t\}$ are not in $\Gamma_{\mathcal{T}}$. Then $A \cup\{\neg s\}$ and $A \cup\{\neg t\}$ are refutable. Hence $A$ can be refuted using $\mathcal{T}_{\neg \wedge}$. Contradiction.

$C_{\forall}$ Let $\forall_{l} s \in A \in \Gamma_{\mathcal{T}}$. Suppose $A \cup\{[s t]\} \notin \Gamma_{\mathcal{T}}$ for some $t \in \mathcal{D} A$ or $A \cup\{[s t]\} \notin$ $\Gamma_{\mathcal{T}}$ for all $t \in \mathrm{EFO}_{\iota}$. Then $A \cup\{[s t]\}$ is refutable for some $t \in \mathcal{D} A$ or $A \cup\{[s t]\}$ is refutable for all $t \in \mathrm{EFO}_{\iota}$. Hence $A$ can be refuted using $\mathcal{T}_{\forall}$.

$C_{\neg \forall} \quad$ Let $\neg \forall_{\iota} s \in A \in \Gamma_{\mathcal{T}}$. Suppose $A \cup\{\neg[s t]\} \notin \Gamma_{\mathcal{T}}$ for every $t \in \mathrm{EFO}_{\iota}$. Then $A \cup\{\neg[s t]\}$ is refutable for every $t \in \mathrm{EFO}_{\iota}$. Hence $A$ is refutable using $\mathcal{T}_{\neg \forall}$ and the finiteness of $A$. Contradiction.

$C_{\mathrm{FE}}$ Let $\left(s \neq \sigma_{\sigma \tau} t\right) \in A \in \Gamma_{\mathcal{T}}$. Suppose $A \cup\{[s x] \neq[t x]\} \notin \Gamma_{\mathcal{T}}$ for every variable $x: \sigma$. Then $A \cup\{[s x] \neq[t x]\}$ is refutable for every $x: \sigma$. Hence $A$ is refutable using $\mathcal{T}_{\mathrm{FE}}$ and the finiteness of $A$. Contradiction.

\section{Theorem 9.2 (Completeness)}

$\mathcal{T}$ and $\mathcal{R}$ can refute every unsatisfiable finite branch.

Proof It suffice to show the claim for $\mathcal{R}$. We prove the claim by contradiction. Let $A$ be an unsatisfiable finite branch that is not refutable. Then $A \in \Gamma_{\mathcal{T}}$ and hence $A$ is satisfiable by Lemmas $9.1,8.2$, and 7.10 .

\section{Compactness and Countable Models}

A branch $A$ is sufficiently pure if for every type $\sigma$ there are infinitely many variables of type $\sigma$ that do not occur in any formula of $A$. Let $\Gamma_{\mathrm{C}}$ be the set of all sufficiently pure branches $A$ such that every finite subset of $A$ is satisfiable. We write $\subseteq_{\mathrm{f}}$ for the finite subset relation.

Lemma 10.1 Let $A \in \Gamma_{\mathrm{C}}$ and $B_{1}, \ldots, B_{n}$ be finite branches such that $A \cup B_{i} \notin \Gamma_{\mathrm{C}}$ for all $i \in\{1, \ldots, n\}$. Then there exists a finite branch $A^{\prime} \subseteq_{\mathrm{f}} A$ such that $A^{\prime} \cup B_{i}$ is unsatisfiable for all $i \in\{1, \ldots, n\}$.

Proof By the assumption, we have for every $i \in\{1, \ldots, n\}$ a finite and unsatisfiable branch $C_{i} \subseteq A \cup B_{i}$. The branch $A^{\prime}:=\left(C_{1} \cup \cdots \cup C_{n}\right) \cap A$ satisfies the claim.

Lemma 10.2 $\Gamma_{\mathrm{C}}$ is a complete abstract consistency class.

Proof We verify the abstract consistency conditions as follows. Lemma 10.1 is used tacitly. 
$C_{\perp}$ We cannot have $\perp \in A$ since $\{\perp\}$ would be an unsatisfiable finite subset.

$C \neg$ We cannot have $\{\neg x, x\} \subseteq A$ since this would be an unsatisfiable finite subset.

$C_{\neq}$We cannot have $(s \neq \imath s) \in A$ since $\{s \neq s\}$ would be an unsatisfiable finite subset.

$C_{\neg \neg}$ Assume $\neg \neg s \in A$ and $A \cup\{s\} \notin \Gamma_{\mathrm{C}}$. There is a finite subset $A^{\prime} \subseteq_{\mathrm{f}} A$ such that $A^{\prime} \cup\{s\}$ is unsatisfiable. There is a model of $A^{\prime} \cup\{\neg \neg s\} \subseteq_{\mathrm{f}} A$. This is also a model of $A^{\prime} \cup\{s\}$, contradicting our choice of $A^{\prime}$.

$C_{\wedge}$ Assume $s \wedge t$ is in $A$ and $A \cup\{s, t\} \notin \Gamma_{\mathrm{C}}$. There is some $A^{\prime} \subseteq_{\mathrm{f}} A$ such that $A^{\prime} \cup\{s, t\}$ is unsatisfiable. There is a model of $A^{\prime} \cup\{s \wedge t\} \subseteq_{\mathrm{f}} A$. This is also a model of $A^{\prime} \cup\{s, t\}$, contradicting our choice of $A^{\prime}$.

$C_{\neg \wedge}$ Assume $\neg(s \wedge t)$ is in $A, A \cup\{\neg s\} \notin \Gamma_{\mathrm{C}}$ and $A \cup\{\neg t\} \notin \Gamma_{\mathrm{C}}$. There is some $A^{\prime} \subseteq_{\mathrm{f}} A$ such that $A^{\prime} \cup\{\neg s\}$ and $A^{\prime} \cup\{\neg t\}$ are unsatisfiable. There is a model of $A^{\prime} \cup\{\neg(s \wedge t)\} \subseteq_{\mathrm{f}} A$. This is also a model of either $A^{\prime} \cup\{\neg s\}$ or $A^{\prime} \cup\{\neg t\}$, contradicting our choice of $A^{\prime}$.

$C_{\mathrm{MAT}}$ Assume $x s_{1} \ldots s_{n}$ and $\neg x t_{1} \ldots t_{n}$ are in $A$ and $A \cup\left\{s_{i} \neq t_{i}\right\} \notin \Gamma_{\mathrm{C}}$ for all $i \in\{1, \ldots, n\}$. There is some $A^{\prime} \subseteq_{\mathrm{f}} A$ such that $A^{\prime} \cup\left\{s_{i} \neq t_{i}\right\}$ is unsatisfiable for all $i \in\{1, \ldots, n\}$. There is a model $\mathcal{I}$ of $A^{\prime} \cup\left\{x s_{1} \ldots s_{n}, \neg x t_{1} \ldots t_{n}\right\} \subseteq_{\mathrm{f}} A$. Since $\hat{\mathcal{I}}\left(x s_{1} \ldots s_{n}\right) \neq \hat{\mathcal{I}}\left(x t_{1} \ldots t_{n}\right)$, we must have $\hat{\mathcal{I}}\left(s_{i}\right) \neq \hat{\mathcal{I}}\left(t_{i}\right)$ for some $i \in$ $\{1, \ldots, n\}$. Thus $\mathcal{I}$ models $A^{\prime} \cup\left\{s_{i} \neq t_{i}\right\}$, contradicting our choice of $A^{\prime}$.

$C_{\text {DEC }}$ Assume $x s_{1} \ldots s_{n} \neq \neq_{\imath} x t_{1} \ldots t_{n}$ is in $A$ and $A \cup\left\{s_{i} \neq t_{i}\right\} \notin \Gamma_{\mathrm{C}}$ for all $i \in$ $\{1, \ldots, n\}$. There is some $A^{\prime} \subseteq_{\mathrm{f}} A$ such that $A^{\prime} \cup\left\{s_{i} \neq t_{i}\right\}$ is unsatisfiable for all $i \in\{1, \ldots, n\}$. There is a model $\mathcal{I}$ of $A^{\prime} \cup\left\{x s_{1} \ldots s_{n} \neq_{\imath} x t_{1} \ldots t_{n}\right\} \subseteq_{\mathrm{f}} A$. Since $\hat{\mathcal{I}}\left(x s_{1} \ldots s_{n}\right) \neq \hat{\mathcal{I}}\left(x t_{1} \ldots t_{n}\right)$, we must have $\hat{\mathcal{T}}\left(s_{i}\right) \neq \hat{\mathcal{I}}\left(t_{i}\right)$ for some $i \in$ $\{1, \ldots, n\}$. Thus $\mathcal{I}$ models $A^{\prime} \cup\left\{s_{i} \neq t_{i}\right\}$, contradicting our choice of $A^{\prime}$.

$C_{\mathrm{BE}} \quad$ Assume $s \neq_{o} t$ is in $A, A \cup\{s, \neg t\} \notin \Gamma_{\mathrm{C}}$ and $A \cup\{\neg s, t\} \notin \Gamma_{\mathrm{C}}$. There is some $A^{\prime} \subseteq_{\mathrm{f}} A$ such that $A^{\prime} \cup\{s, \neg t\}$ and $A^{\prime} \cup\{\neg s, t\}$ are unsatisfiable. There is a model of $A^{\prime} \cup\left\{s \neq_{o} t\right\} \subseteq_{\mathrm{f}} A$. This is also a model of $A^{\prime} \cup\{s, \neg t\}$ or $A^{\prime} \cup\{\neg s, t\}$.

$C_{\mathrm{FE}}$ Assume $s \neq_{\sigma \tau} t$ is in $A$. Since $A$ is sufficiently pure, there is a variable $x: \sigma$ which does not occur in $A$. Assume $A \cup\{[s x] \neq[t x]\} \notin \Gamma_{\mathrm{C}}$. There is some $A^{\prime} \subseteq_{\mathrm{f}} A$ such that $A^{\prime} \cup\{[s x] \neq[t x]\}$ is unsatisfiable. There is a model $\mathcal{I}$ of $A^{\prime} \cup\{s \neq t\} \subseteq_{\mathrm{f}} A$. Since $\hat{\mathcal{I}}(s) \neq \hat{\mathcal{T}}(t)$, there must be some $a \in \mathcal{I} \sigma$ such that $\hat{\mathcal{I}}(s) a \neq \hat{\mathcal{I}}(t) a$. Since $x$ does not occur in $A$, we know $\widehat{\mathcal{I}_{a}^{x}}(s x) \neq \widehat{\mathcal{I}_{a}^{x}}(t x)$ and $\mathcal{I}_{a}^{x}$ is a model of $A^{\prime}$. Since $\widehat{\mathcal{I}_{a}^{x}}([s x])=\widehat{\mathcal{I}_{a}^{x}}(s x)$ by N4 and $\widehat{\mathcal{I}_{a}^{x}}([t x])=\widehat{\mathcal{I}_{a}^{x}}(t x)$, we conclude $\mathcal{I}_{a}^{x}$ is a model of $A^{\prime} \cup\{[s x] \neq[t x]\}$, contradicting our choice of $A^{\prime}$.

$C_{\text {Con }}$ Assume $s=\imath t$ and $u \neq \imath, v$ are in $A, A \cup\{s \neq u, t \neq u\} \notin \Gamma_{\mathrm{C}}$ and $A \cup\{s \neq$ $v, t \neq v\} \notin \Gamma_{\mathrm{C}}$. There is some $A^{\prime} \subseteq_{\mathrm{f}} A$ such that $A^{\prime} \cup\{s \neq u, t \neq u\}$ and $A^{\prime} \cup\{s \neq v, t \neq v\}$ are unsatisfiable. There is a model $\mathcal{I}$ of $A^{\prime} \cup\{s=t, u \neq$ 
$v\} \subseteq_{\mathrm{f}} A$. Since $\hat{\mathcal{I}}(s)=\hat{\mathfrak{I}}(t)$ and $\hat{\mathcal{I}}(u) \neq \hat{\mathcal{T}}(v)$, we either have $\hat{\mathfrak{T}}(s) \neq \hat{\mathcal{I}}(u)$ and $\hat{\mathcal{I}}(t) \neq \hat{\mathcal{I}}(u)$ or $\hat{\mathcal{I}}(s) \neq \hat{\mathcal{I}}(v)$ and $\hat{\mathfrak{I}}(t) \neq \hat{\mathcal{I}}(v)$. Hence $\mathcal{I}$ models either $A^{\prime} \cup\{s \neq u, t \neq u\}$ and $A^{\prime} \cup\{s \neq v, t \neq v\}$, contradicting our choice of $A^{\prime}$.

$C_{\forall}$ Assume $\forall_{l} s$ is in $A$ and $A \cup\{[s t]\} \notin \Gamma_{C}$ for some $t \in \mathrm{EFO}_{l}$. There is some $A^{\prime} \subseteq_{\mathrm{f}} A$ such that $A^{\prime} \cup\{[s t]\}$ is unsatisfiable. There is a model $\mathcal{I}$ of $A^{\prime} \cup\left\{\forall_{l} s\right\} \subseteq_{\mathrm{f}} A$. Note that $\hat{\mathcal{I}}([s t])=\hat{\mathcal{I}}(s t)=\hat{\mathcal{I}}(s)(\hat{\mathcal{I}}(t))=1$ using N4. Hence $\mathcal{I}$ is a model of $A^{\prime} \cup\{[s t]\}$, contradicting our choice of $A^{\prime}$.

$C_{\neg \forall}$ Assume $\neg \forall_{\iota} s$ is in $A$. Since $A$ is sufficiently pure, there is a variable $x_{\iota}$ which does not occur in $A$. Assume $A \cup\{\neg[s x]\} \notin \Gamma_{\mathrm{C}}$. There is some $A^{\prime} \subseteq_{\mathrm{f}} A$ such that $A^{\prime} \cup\{\neg[s x]\}$ is unsatisfiable. There is a model $\mathcal{I}$ of $A^{\prime} \cup\left\{\neg \forall_{l} s\right\} \subseteq_{\mathrm{f}} A$. There is some $a \in \mathcal{I} \iota$ such that $\hat{\mathcal{I}}(s) a=0$. Since $x$ does not occur in $A$, we know $\widehat{\mathcal{I}_{a}^{x}}(s) a=\widehat{\mathcal{I}}(s) a=0$ and $\mathcal{I}_{a}^{x}$ is a model of $A^{\prime}$. Note that $\widehat{\mathcal{I}_{a}^{x}}([s x])=\widehat{\mathcal{I}_{a}^{x}}(s x)=\widehat{\mathcal{I}_{a}^{x}}(s) a=0$ using N4. Hence $\mathcal{I}$ is a model of $A^{\prime} \cup\{\neg[s x]\}$, contradicting our choice of $A^{\prime}$.

We show the completeness of $\Gamma_{\mathrm{C}}$ by contradiction. Let $A \in \Gamma_{\mathrm{C}}$ and $s, t \in \mathrm{EFO}_{\iota}$ such that $A \cup\{[s=t]\}$ and $A \cup\{[s \neq t]\}$ are not in $\Gamma_{\mathrm{C}}$. Then there exists $A^{\prime} \subseteq_{\mathrm{f}} A$ such that $A^{\prime} \cup\{[s=t]\}$ and $A^{\prime} \cup\{[s \neq t]\}$ are unsatisfiable. Contradiction by N4 since $A^{\prime}$ is satisfiable.

\section{Theorem 10.3 (Compactness)}

A branch is satisfiable if each of its finite subsets is satisfiable.

Proof Let $A$ be a branch such that every finite subset of $A$ is satisfiable. Without loss of generality we assume $A$ is sufficiently pure. Then $A \in \Gamma_{\mathrm{C}}$. Hence $A$ is satisfiable by Lemmas $10.2,8.2$, and 7.10 .

\section{Theorem 10.4 (Countable Models)}

Every satisfiable branch has a countable model.

Proof Let $A$ be a satisfiable branch. Without loss of generality we assume that $A$ is sufficiently pure. Hence $A \in \Gamma_{\mathrm{C}}$. By Lemmas 10.2 and 8.2 we have a complete evident set $E$ such that $A \subseteq E$. By Lemma 7.10 we have a countable model for $E$ and hence for $A$.

Theorem 10.5 (Countable Model Existence)

Every evident branch has a countable model.

Proof Let $E$ be an evident branch. By Lemma 7.10 we know that $E$ is satisfiable. By Theorem 10.4 we know that $E$ has a countable model. 


\section{Decidability}

The tableau system $\mathcal{R}$ defined in $\$ 9$ yields a procedure that decides the satisfiability of three substantial fragments of EFO. Starting with the initial branch, the procedure applies tableau rules until it reaches a branch that contains $\perp$ or cannot be extended with the tableau rules. The procedure returns "satisfiable" if it arrives at a terminal branch that does not contain $\perp$, and "unsatisfiable" if it finds a refutation. There are branches on which the procedure does not terminate (e.g., $\{\forall, x . f x \neq x\})$. We first establish the partial correctness of the procedure.

Proposition 11.1 (Verification Soundness) Let $A$ be a finite branch that does not contain $\perp$ and cannot be extended with $\mathcal{R}$. Then $A$ is evident and has a finite model.

Proof The evidence of a branch as specified is easily verified. The existence of a finite model follows with Theorem 7.9 .

\section{Proposition 11.2 (Refutation Soundness)}

Every refutable branch is unsatisfiable.

Proof Let the branches $A_{1}, \ldots, A_{n}$ be obtained from a satisfiable branch $A$ by application of a rule of $\mathcal{R}$. It suffices to show that one of the branches $A_{1}, \ldots, A_{n}$ is satisfiable. For $\mathcal{T}_{\neg}$ this follows from the fact that the implication $x \wedge \neg x \rightarrow \perp$ is valid. For $\mathcal{T}_{\neg \wedge}$ the validity of $\neg(x \wedge y) \rightarrow \neg x \vee \neg y$ suffices, and for $\mathcal{T}_{\mathrm{FE}}$ the validity of $f \neq g \rightarrow \exists x . f x \neq g x$ does the job. The soundness of the other rules follows with similar arguments.

For the termination of the procedure we consider the relation $A \rightarrow A^{\prime}$ that holds if $A$ and $A^{\prime}$ are branches such that $\perp \notin A \subsetneq A^{\prime}$ and $A^{\prime}$ can be obtained from $A$ by applying a rule of $\mathcal{R}$. We say that $\mathcal{R}$ terminates on a set $\Delta$ of branches if there is no infinite derivation $A \rightarrow A^{\prime} \rightarrow A^{\prime \prime} \rightarrow \cdots$ such that $A \in \Delta$.

Proposition 11.3 Let $\mathcal{R}$ terminate on a set $\Delta$ of finite branches. Then satisfiability of the branches in $\Delta$ is decidable and every satisfiable branch in $\Delta$ has a finite model.

Proof Follows with Propositions 11.2 and 11.1 and Theorem 7.9 ,

The decision procedure depends on the normalization operator employed with $\mathcal{R}$. A normalization operator that yields $\beta$-normal forms provides for all termination results proven in this section. Note that the tableau system applies the normalization operator only to applications $s t$ where $s$ and $t$ are both normal and $t$ has type $\iota$ if it is not a variable. Hence at most one $\beta$-reduction is needed 
for normalization if $s$ and $t$ are $\beta$-normal. Moreover, no $\alpha$-renaming is needed if the bound variables are chosen differently from the free variables. For clarity, we continue to work with an abstract normalization operator and state a further condition:

N5 The least relation $\succ$ on terms such that

1. $a s_{1} \ldots s_{n} \succ s_{i}$ if $i \in\{1, \ldots, n\}$

2. $s>[s x]$ if $s: \sigma \tau$ and $x: \sigma$

terminates on normal terms.

\subsection{Pure Disequations}

A type is pure if it does not contain $o$. A term is pure if the type of every name occurring in it (bound or unbound) is pure. An equation $s=t$ or disequation $s \neq t$ is pure if $s$ and $t$ are pure terms.

Proposition 11.4 (Pure Termination) Let the normalization operator satisfy N5. Then $\mathcal{R}$ terminates on finite branches containing only pure disequations.

Proof Let $A \rightarrow A_{1} \rightarrow A_{2} \rightarrow \cdots$ be a possibly infinite derivation that issues from a finite branch containing only pure disequations. Then no other rules but possibly $\mathcal{T}_{\mathrm{DEC}}, \mathcal{R}_{\mathrm{FE}}$, and $\mathcal{T}_{\neq}$apply and thus no $A_{i}$ contains a formula that is not $\perp$ or a pure disequations (using S5). Using N5 it follows that the derivation is finite.

We now know that the validity of pure equations is decidable, and that the invalidity of pure equations can be demonstrated with finite interpretations (Proposition [11.1]. Both results are well-known [6, 10], but it is remarkable that we obtain them with different proofs and as a byproduct.

Example 11.5 $\mathcal{R}$ does not terminate on branches that contain pure equations and pure disequations. We assume the typing $F:(\iota \iota) \iota$ and $f: \iota \iota$.

$$
\begin{array}{ll}
F(\lambda x \cdot F(f x))=a, \quad F(f a) \neq a & \text { initial branch } \\
F(\lambda x \cdot F(f x)) \neq F(f a), \quad a \neq F(f a) & \mathcal{T}_{\mathrm{CON}} \\
(\lambda x \cdot F(f x)) \neq f a & \mathcal{T}_{\mathrm{DEC}} \\
F(f b) \neq f a b & \mathcal{R}_{\mathrm{FE}} \\
F(\lambda x \cdot F(f x)) \neq F(f b), \quad a \neq F(f b) & \mathcal{T}_{\mathrm{CON}}
\end{array}
$$

The non-termination depends on the fact that the positive equation is not firstorder. We can prove termination if we constrain the positive equations to be first-order, that is, to contain only variables whose type has the form $\iota .$. . . This 
restriction yields $\mathcal{R}_{\mathrm{FE}}$ non-applicable. Admitting quantifier-free formulas with variables whose types have the form $\sigma_{1} \ldots \sigma_{n} o$ where $n \geq 0$ and $\sigma_{1}, \ldots, \sigma_{n}$ are pure types also preserves termination.

\subsection{Bernays-Schönfinkel-Ramsey Formulas}

It is well-known that satisfiability of Bernays-Schönfinkel-Ramsey formulas (firstorder $\exists^{*} \forall^{*}$-prenex formulas without functions) is decidable and the fragment has the finite model property [4]. We reobtain this result by showing that $\mathcal{R}$ terminates for the respective fragment. We call a type BSR if it is $\iota$ or $o$ or has the form $\iota \ldots \iota$. We call an EFO formula $s$ BSR if it satisfies two conditions:

1. The type of every variable that occurs in $s$ is BSR.

2. $\forall$, does not occur below a negation in $s$.

For simplicity, our BSR formulas don't provide for outer existential quantification. We need one more condition for the normalization operator:

N6 If $s: \iota 0$ is BSR and $x: \iota$, then $[s x]$ is BSR.

Proposition 11.6 (BSR Termination) Let the normalization operator satisfy N5 and N6. Then $\mathcal{R}$ terminates on finite branches containing only BSR formulas.

Proof Let $A \rightarrow A_{1} \rightarrow A_{2} \rightarrow \cdots$ be a possibly infinite derivation that issues from a finite branch containing only BSR formulas. Then $\mathcal{R}_{\neg \forall}$ and $\mathcal{R}_{\mathrm{FE}}$ are not applicable and all $A_{i}$ contain only BSR formulas (using N6). Furthermore, at most one new variable is introduced. Since all terms of type $\iota$ are variables, there is only a finite supply. Using N5 it follows that the derivation is finite.

\subsection{Lambda-Free Formulas}

In [3] we study lambda- and quantifier-free EFO and show that the concomitant subsystem of $\mathcal{R}$ terminates on finite branches. The result extends to lambda-free branches containing quantifiers (e.g., $\left\{\forall_{l} f\right\}$ ).

Proposition 11.7 (Lambda-Free Termination) Let the normalization operator satisfy $[s]=s$ for every lambda-free EFO term $s$. Then $\mathcal{R}$ terminates on finite lambda-free branches.

Proof An application of $\mathcal{R}_{\mathrm{FE}}$ disables a disequation $s \neq_{\sigma \tau} t$ and introduces new subterms as follows: a variable $x: \sigma$, two terms $s x: \tau$ and $t x: \tau$, and two formulas $s x=t x$ and $s x \neq t x$. Since the types of the new subterms are smaller than the type of $s$ and $t$, and the new subterms introduced by the other rules always have type $o$ or $\iota$, no derivation can employ $\mathcal{R}_{\mathrm{FE}}$ infinitely often. 
Let $A \rightarrow A_{1} \rightarrow A_{2} \rightarrow \cdots$ be a possibly infinite derivation that issues from a finite lambda-free branch and does not employ $\mathcal{R}_{\mathrm{FE}}$. It suffices to show that the derivation is finite. Observe that no new subterms of the form $\forall_{l} s$ are introduced. Hence only finitely many new subterms of type $\iota$ are introduced. Consequently, only finitely many new subterms of type $o$ are introduced. Hence the derivation is finite.

\section{Conclusion}

In this paper we have shown that the EFO fragment of Church's type theory enjoys the characteristic properties of first-order logic. We have devised a complete tableau system that comes with a new treatment of equality (confrontation) and a novel subterm restriction for the universal quantifier (discriminating terms). The tableau system decides lambda-free formulas, Bernays-Schönfinkel-Ramsey formulas, and equations between pure lambda terms.

\section{References}

[1] Peter B. Andrews. Classical type theory. In Alan Robinson and Andrei Voronkov, editors, Handbook of Automated Reasoning, volume 2, chapter 15, pages 965-1007. Elsevier Science, 2001.

[2] Chad E. Brown. Automated Reasoning in Higher-Order Logic: Set Comprehension and Extensionality in Church's Type Theory. College Publications, 2007.

[3] Chad E. Brown and Gert Smolka. Terminating tableaux for the basic fragment of simple type theory. In M. Giese and A. Waaler, editors, TABLEAUX 2009, volume 5607 of LNCS (LNAI), pages 138-151. Springer, 2009.

[4] Egon Börger, Erich Grädel, and Yuri Gurevich. The Classical Decision Problem. Springer, 1997.

[5] Melvin Fitting. First-Order Logic and Automated Theorem Proving. Springer, 1996.

[6] Harvey Friedman. Equality between functionals. In R. Parikh, editor, Proc. Logic Colloquium 1972-73, volume 453 of Lectures Notes in Mathematics, pages 22-37. Springer, 1975.

[7] J. R. Hindley. Basic Simple Type Theory, volume 42 of Cambridge Tracts in Theoretical Computer Science. Cambridge University Press, 1997. 
[8] Dag Prawitz. Hauptsatz for higher order logic. J. Symb. Log., 33:452-457, 1968.

[9] Raymond M. Smullyan. First-Order Logic. Springer, 1968.

[10] Richard Statman. Completeness, invariance and lambda-definability. $J$. Symb. Log., 47(1):17-26, 1982. 